\title{
Endoscopic submucosal dissection in the treatment of patients with early colorectal carcinoma and precancerous lesions
}

\author{
Jing Yu, Yan Zhang, Junbo Qian \\ Department of Gastroenterology, the Second Affiliated Hospital of Nantong University, Nantong, China \\ Contributions: (I) Conception and design: J Yu; (II) Administrative support: J Qian; (III) Provision of study materials or patients: J Qian; (IV) \\ Collection and assembly of data: J Yu; (V) Data analysis and interpretation: J Yu; (VI) Manuscript writing: All authors; (VII) Final approval of \\ manuscript: All authors. \\ Correspondence to: Junbo Qian. Department of Gastroenterology, the Second Affiliated Hospital of Nantong University, 6 Hai'er Xiang North Road, \\ Nantong 226001, China. Email: ntyyqjb@163.com.
}

\begin{abstract}
Background: Our study aims to explore the indications and clinical efficacy of endoscopic submucosal dissection (ESD) on the early colorectal carcinoma and precancerous lesions.

Methods: The clinical data of 29 patients with early colorectal carcinoma and precancerous lesions who were treated with ESD at Nantong First People's Hospital between January 2018 and December 2019 were collected. Then the endoscopic morphology, postoperative pathological classification, tumor resection rate, postoperative complications, and follow-up outcomes were analyzed.

Results: Colorectal carcinoma lesions were distributed in the left colon, accounting for $89.6 \%$. There were 14 cases $(48.3 \%)$ with protuberant endoscopic tumors, accounting for the highest proportion, while 2 cases $(6.9 \%)$ of the flat tumors, accounting for the lowest proportion. The average operation time for ESD was 123 minutes, and en-bloc resection was $100 \%$ while the curative resection rate was $89.6 \%$. There were 3 cases $(10.3 \%)$ with delayed hemorrhage after ESD, and 1 case with persistent hemorrhage during the operation was transferred to surgical treatment. No cases with infection or perforation after ESD. For postoperative pathological classification, villous-tubular adenoma with low-grade epithelioma accounted for 31\%; tubular adenoma with high-grade epithelioma only accounted for $3.4 \%$. There was no recurrence in the follow-up for 1-20 months.
\end{abstract}

Conclusions: Control of surgical indications strictly, improvement of operation skills, attention to postoperative pathological feedback, and close follow-up are necessary guarantees to improve the clinical effectiveness of ESD.

Keywords: Endoscopic submucosal dissection (ESD); early colorectal carcinoma and precancerous lesions; clinical experience

Submitted Aug 31, 2020. Accepted for publication Oct 12, 2020.

doi: 10.21037/jgo-20-393

View this article at: http://dx.doi.org/10.21037/jgo-20-393

\section{Introduction}

As the aging of the world population and changes in eating habits lead to obesity and reduced exercise, colorectal carcinoma has become the fourth deadly cancer in the world that threatens human health (1). Early diagnosis of colorectal carcinoma and prompt intervention can even cure the patients at the early stage, while the efficacy is low for advanced patients (2). Compared with the traditional surgical treatment of colorectal cancer, the current treatment of early colorectal cancer is more biased towards minimally invasive treatment. Ferlitsch believes that endoscopic resection is a viable option for precancerous lesions and T1 cancer (3); the research of Raju and Law found that endoscopic tumor resection is safer than surgical operation (4,5); Fuccio et al. found that after standard ESD The 12 -month recurrence rate is low, about $2 \%$, and it is 
found that the recurrence rate in Asian countries (1\%) is lower than that in Western countries (5\%) (6).

Endoscopic submucosal dissection (ESD) is developed on the basis of endoscopic mucosal resection (EMR) as a new and efficient endoscopic minimally invasive technique, the treatment is mainly for early digestive tract cancer and precancerous lesions. At present, it is more and more widely used in the diagnosis and treatment of early esophageal cancer and gastric cancer $(7,8)$. The method is based on the endoscopic submucosal injection, using several special high-frequency electric knives to peel off the mucosa where the lesion is located to achieve the purpose of treatment. The pathological changes can be completely removed through ESD to achieve the effect of radical treatment of gastrointestinal tumors. ESD is also a standard treatment for early colorectal carcinoma with almost no risk of lymph node metastasis $(9,10)$, which can provide a high resection rate and contribute to the evaluation of accurate histopathology $(11,12)$. This study retrospectively analyzed the clinical data of 29 patients with early colorectal carcinoma and precancerous lesions who received ESD treatment to explore the safety and efficacy of ESD in the treatment of colorectal carcinoma and precancerous lesions, the postoperative review adopts Linked Color Imaging (LCI) and Blue Laser Imaging (BLI, Blue Laser Imaging) technologies, which at the same time extend the followup time of patients and improve the accuracy of the review Therefore, thus providing a better theoretical basis for the clinical application of ESD. We present the following article in accordance with the STROBE reporting checklist (available at http://dx.doi.org/10.21037/jgo-20-393).

\section{Methods}

\section{General data}

The clinical data were collected from 29 patients with early colorectal cancer and precancerous lesions who were treated with ESD in the Endoscopy Center of the Department of Gastroenterology, Nantong First People's Hospital between January 2018 and December 2019. There were 15 males and 14 females, aged $41-80$ years (average age: 63 years). On admission, the patients presented primarily with abdominal pain and distension, bloody stool, changes in stool character, and some were asymptomatic physical examinees. This study was approved by the Ethics Committee of the Second Affiliated Hospital of Nantong University (No. 2019KS095). All procedures performed in this study involving human participants were in accordance with the Declaration of Helsinki (as revised in 2013). Individual consent for this retrospective analysis was waived.

\section{Endoscopic therapy}

\section{Main devices}

Olympus CF-H290I colonoscope, ERBE high-frequency resection device, APC 300 argon plasma coagulator, Olympus KD-650Q hook knife, Medical-Globe GmbH GSN-05-19-230 injection needle, transparent cap, hot biopsy forceps, ROCC-D-26-195-C hemostatic clip, and others.

\section{Preoperative preparation}

Blood routine, coagulation function, infectious markers, and abdominal CT and other preoperative examinations; bowel preparation using conventional polyethylene glycolelectrolyte powder.

\section{ESD operation steps}

(I) Marking: the boundary of the lesion was identified, and about $5 \mathrm{~mm}$ around the periphery of the lesion was marked after staining with indigo carmine. (II) Submucosal injection: submucosal injection is performed at multiple points lateral to the marker points to lift the lesion and separate it from the muscular layer. (III) Incision of the tissue around the lesion: the mucosa was incised circumferentially around the lesion using a single-use Dualknife, and then the submucosa was separated along the muscularis propria surface. The incision was started from the distal end of the lesion. (IV) Submucosal dissection: the tumor is dissected layer by layer. (V) Wound management: observed the wound and stopped hemorrhage in time.

\section{Efficacy criteria}

For patients with early colorectal cancer and precancerous lesions treated with ESD, the resection margin, lymph node, and distant metastasis of postoperative specimens were essential to test radical resection. Meanwhile, the pathology of postoperative specimens was pivotal. If the adenoma and intramucosal cancer with a negative margin, it was a radical resection. The intramucosal cancer with positive margins, and colorectal cancer with SM1 invasion, should be tested more carefully to determine whether radical resection had been performed, and additional surgical intervention might be required. 
Table 1 Mass distribution and endoscopic morphology of 29 patients with early colorectal cancer and precancerous lesions

\begin{tabular}{|c|c|c|c|c|c|c|c|}
\hline $\begin{array}{l}\text { Endoscopic } \\
\text { morphology }\end{array}$ & Case & \multicolumn{4}{|c|}{ Left colon } & \multicolumn{2}{|c|}{ Right colon } \\
\hline Protuberant & 14 & 8 & 4 & 1 & 0 & 1 & 0 \\
\hline Flat & 2 & 2 & 0 & 0 & 0 & 0 & 0 \\
\hline Literally spreading & 13 & 9 & 0 & 0 & 1 & 1 & 2 \\
\hline
\end{tabular}

\section{Postoperative management}

The patients fasted for about 24-48 hours after the operation. Their vital signs were studied, and the patients were given an asymptomatic, fluid replacement, antiinfective treatment simultaneously. Prolonged observation time and endoscopic treatment are given according to changes in the patients' condition.

\section{Follow-up}

At 1, 3, 6, 12, and 20 months after the operation, regular outpatient appointments were made for follow-up. Tumor index detection observed the recurrence and endoscopic reexamination.

\section{Results}

\section{Detection of patients with early colorectal cancer and} precancerous lesions after ESD

There were 10 and 19 patients underwent intestinal ESD in 2018 and 2019, respectively. Seven and 19 patients were detected with early colorectal cancer and precancerous lesions, accounting for about $24.2 \%$ and $65.5 \%$, respectively. Another 3 patients (10.3\%) had other surgeries because of carcinogenesis.

\section{Characteristics of early colorectal cancer and precancerous lesions}

In the 29 patients treated with ESD, the size and diameter of the lesions were $0.8-4.5 \mathrm{~cm}$, the extent of the lesions was $0.8 \times 0.8-4.5 \times 6 \mathrm{~cm}$, and the average size was $2.0 \times 2.6 \mathrm{~cm}$. There were 25 patients $(86.2 \%)$ with left colon lesions, 19 $(65.5 \%)$ in the rectum, $4(13.8 \%)$ in the sigmoid colon, 1 $(3.4 \%)$ in the descending colon, and $1(3.4 \%)$ in the spleen. There were four patients (13.8\%) with lesions in the right colon, including $2(6.9 \%)$ in the transverse colon, $2(6.9 \%)$ in the ascending colon.

There were 14 cases $(48.3 \%)$ with protuberant tumors, 13 cases $(44.8 \%)$ with laterally spreading tumors, and 2 cases $(6.9 \%)$ with flat tumors. Among the patients, the highest proportion was villous tubular adenoma with lowgrade intraepithelial neoplasia in 9 cases $(31 \%)$, while the lowest proportion was tubular adenoma with high-grade intraepithelial neoplasia 1 case (3.4\%). The characteristics of colorectal cancer lesions in 29 patients are shown in Table 1.

\section{Efficacy of ESD treatment}

Twenty-eight patients completed ESD, while the other one case (lesion in the rectum) was directly transferred to surgery therapy because of frequent hemorrhage and local adhesion during the ESD, who was diagnosed as moderately differentiated adenocarcinoma of the rectum by postoperative pathological detection. All 29 patients were followed up and reexamined using linked color imaging (LCI) and blue laser imaging (BLI) 12 months after ESD (Figure 1), with an average operation time of 123 minutes. The follow-up result showed that 26 cases $(89.6 \%)$ were cured. One case (lesion in the rectum) was tubular-villous adenoma, pathologically confirmed as moderately differentiated tubular adenocarcinoma with infiltration after resection margin, who was transferred to surgery. Another 1 case (lesion in the descending colon) is a well-differentiated tubular adenocarcinoma with SM2 infiltration (infiltrating the deep submucosal layer), followed by additional surgery.

\section{Complications}

All patients had different degrees of hemorrhage during operation but were successfully stopped using hot biopsy forceps, argon knife, and titanium clips under the 

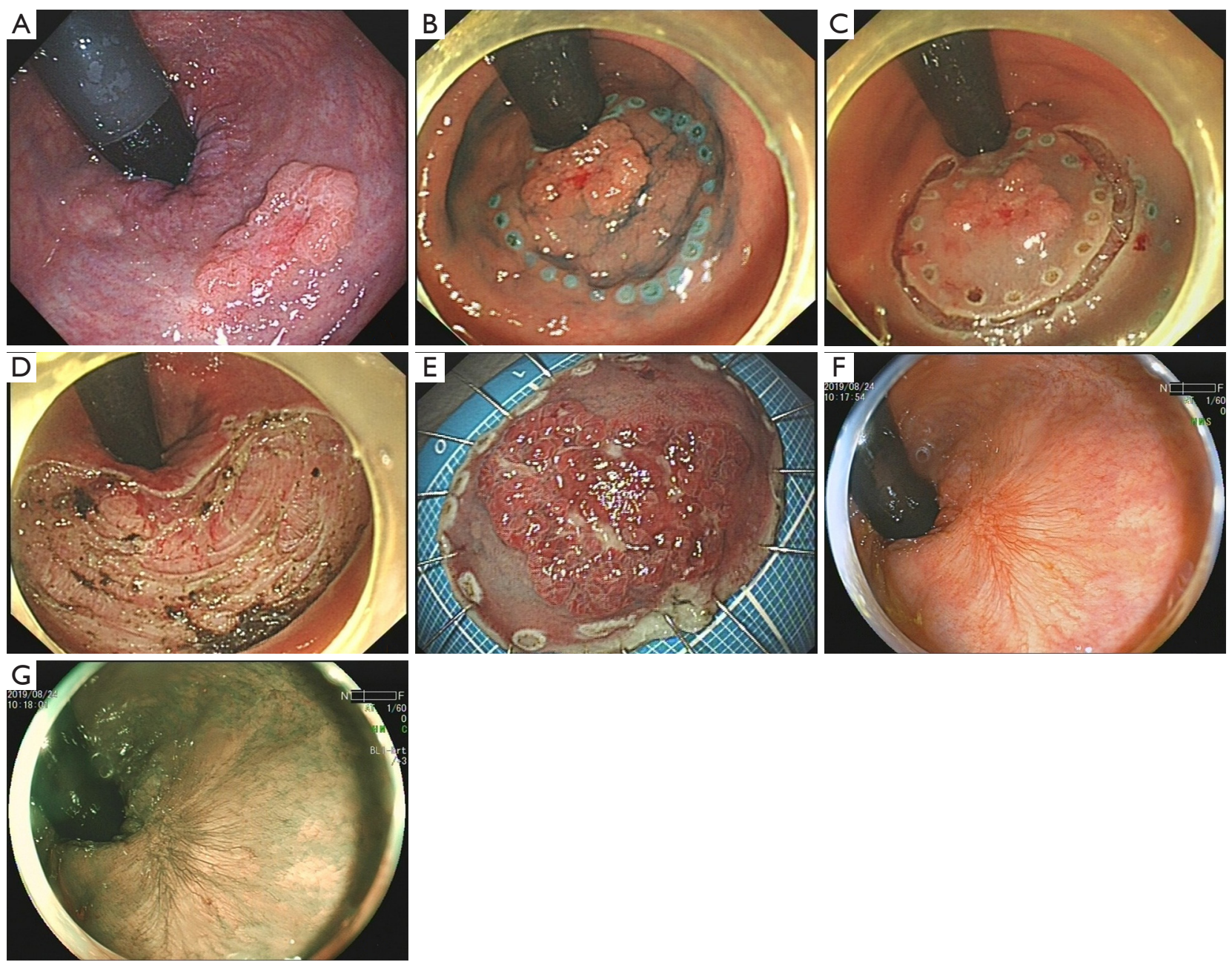

Figure 1 Endoscopic submucosal dissection (ESD) treatment and postoperative review of rectal mucosal protuberant lesions. A male patient, 60 years old. (A) $2.0 \mathrm{~cm} \times 1.0 \mathrm{~cm}$ mass was observed under the endoscope; (B) $0.5 \mathrm{~cm}$ outside the edge of indigo carmine staining was marked; (C) the marked points were first separated outwards with a single-use Dualknife, and then flossing traction stripped the mucosa; (D) the wound surface was treated with the hot biopsy forceps; (E) the specimen was paved and soaked in formaldehyde. Its postoperative pathology shows villous tubular adenoma with low-grade epithelial neoplasia with negative horizontal resection margins. (F,G) After 12 months, no abnormal vessels were observed under linked color imaging (LCI) and blue laser imaging (BLI).

endoscope. Delayed postoperative hemorrhage occurred in 3 patients $(10.3 \%)$ within 48 hours after ESD. The peak period of hemorrhage was at 48 hours after surgery, which might be related to the extensive surgical wounds and insufficient hemostasis during the operation. There is no postoperative perforation and fever in the patients.

\section{Follow-up outcomes}

The patients made appointments in the outpatient department for reexamination, with a period of about
1-20 months (median time: 6.8 months). At present, all patients recovered well during follow-up, and no recurrence was observed.

\section{Discussion}

Colorectal carcinoma is a common malignant tumor. With the development of the Chinese economy and changes in people's lifestyles, the incidence and mortality of colorectal carcinoma are also on the rise. In 2015, there were 376,000 recent cases and 191,000 deaths (13). The survival rate of 
patients with colorectal carcinoma at a progressive and advanced stage is low. Endoscopic intervention for early colorectal carcinoma and precancerous lesions might significantly improve the survival rate and quality of life of patients $(14,15)$. With the development of endoscopic technology, modern technologies have been widely used, including endoscopic ultrasonography, chromoendoscopy, and high-resolution digital chromoendoscopy. ESD not only has applied in more indications but also has a greater improvement in safety, which has become an important approach to screen and treat the early colorectal carcinoma and precancerous lesions (16). However, complications like delayed hemorrhage, perforation, and infection often occur after colorectal ESD. The complications not only prolong the hospitalization of patients but also require added surgery sometimes, increasing the economic burden of patients and consuming additional medical resources. Therefore, this study aimed to investigate the efficacy and safety of ESD in the treatment of early colorectal carcinoma and precancerous lesions.

The detection rates of early colorectal carcinoma and precancerous lesions in the endoscopy center of our hospital in 2018 and 2019 were $30.3 \%$ and $40.5 \%$, respectively. This increasing trend, on the one hand, was related to changes in diet, emotion, living environment, and a Westernized lifestyle. However, it was associated with the improvement and popularization of endoscopic technology and changes of endoscopists' concept for early colorectal carcinoma and precancerous lesions. In this clinical study, we found that a high proportion of early carcinoma occurred in the left colon like the rectum, sigmoid, and the descending colon, accounting for $86.2 \%$. Among the precancerous lesions in the 29 patients, the primary histological type was villous tubular adenoma with low-grade intraepithelial neoplasia, with 9 cases $(31 \%)$. And the primary histological type of patient with early colorectal carcinoma was adenoma with intramucosal carcinogenesis, with 7 cases (24.1\%). In this study, protuberant and laterally spreading tumors accounted for the majority, showing that depressed lesions were easily ignored under endoscopy. However, the depressed lesions have a high tendency of malignant transformation, so endoscopists need to improve the awareness and ability to identify depressed early colorectal carcinoma and its precancerous lesions.

ESD in the treatment colonic submucosal lesions aims for radical resection, so strict and careful selection of indications is needed. The lymph node metastasis was determined according to the consensus on screening, diagnosis, and treatment of early colorectal carcinoma and precancerous lesions in China and the latest intestinal ESD guidelines developed by the Japanese Society of Gastrointestinal Endoscopy in the second edition of the ESD/EMR guidelines for colorectal lesions in 2019. Tumor size and location are used to determine whether the tumor can be resected entirely; colorectal adenoma and intramucosal cancer are absolute indications. However, the treatment options for patients with SM1 invasion (submucosal invasion depth of $1 \mathrm{~mm}$ ) need to be cautious (17). Among 29 patients in this study, there were 12 cases with postoperative pathological low-grade epithelial neoplasia, 7 cases with high-grade epithelial neoplasia, and 7 cases with adenoma accompanied by focal carcinogenesis. The patients with no regional lymph node metastasis underwent radical resection at one time. However, there were 2 cases of moderately differentiated adenocarcinoma and 1 case of well-differentiated adenocarcinoma, with SM1 invasion. Added surgery was needed to ensure the therapeutic effect of the three patients. It is especially important to test the specimens resected after intestinal ESD, which are not only needed to determine the pathological tissues but also provide the data of infiltration depth, mucosal level, vascular infiltration, and vertical resection margin status. According to the latest Japanese endoscopic guidelines, endoscopic treatment was performed for tumor masses with a diameter $\leq 5.0 \mathrm{~cm}$ and without blood and lymphatic metastasis. In this study, the average size of the masses was about $2.0 \mathrm{~cm} \times$ $2.6 \mathrm{~cm}$, and the maximum length was $6.0 \mathrm{~cm}$, so the lesions were relatively easy to be dissected entirely. Among the seven patients with local cancer lesions, two patients had an invasion of the deep submucosal layer, who were needed strict evaluation whether endoscopic treatment was applied.

Hemorrhage and perforation after ESD are common complications (18). Ogasawara et al. (19) have concluded that rectal lesions can be used as an independent risk factor for delayed hemorrhage after rectal ESD, and the closer the lesion is to the anus, the easier it is to bleed. In the present study, ESD was performed on 29 patients. There was 1 case of persistent intraoperative hemorrhage, and the lesion was in the rectum; because of the double blood supply of the inferior rectal artery and perianal artery, it was easy to bleed during the operation and difficult to control, so the low colorectal lesions should be thoroughly evaluated before the operation. There were 3 cases of delayed hemorrhage after the operation, but no cases of perforation. The reason of the low incidence of complications in this study was: first, strict control of surgical indications; second, fully preoperative 
assessment of the size and depth of the mass, and the following conditions were considered: the lesions were mostly in the sigmoid and rectum, and the lesion locations were not deep, the endoscope was easy to reach the lesions; the focus was fully exposed, and the accurate operation was performed under a good field of view and operation space; third, preparation of various plans during and after surgery for timely treatment.

Endoscopic treatment is safe and effective for early colorectal cancer. ESD combined with other technical means can further reduce complications. Before the ESD operation, the ultrasound endoscopy (EUS) and blue laser image (BLI) are used to assess the tumor invasion depth and superficial microstructure, which can enable the surgeon to more accurately grasp the indications for the operation; for a mass of $2.5 \mathrm{~cm}$ in diameter, ESD combined with ferrule technology can not only greatly shorten the operation time, but also greatly reduce complications such as intraoperative and postoperative bleeding and perforation. Compared with electrosurgical knives, laser surgical knives can generate less heat during mucosal incision and separation. The ESD operation generates less heat energy and greatly reduces the probability of intestinal perforation.

At present, there is an insufficient strict standard process for the follow-up after ESD in the treatment of early colorectal carcinoma, resulting in the loss of followup sometimes, which affects the complete understanding of the postoperative progress of the disease. The Japanese guidelines mentioned earlier recommended follow-up at 1 , 3,6 , and 12 months after endoscopic surgery, with dynamic detection of tumor indicators and routine endoscopic review. In this study, patients were followed up for 1-20 months. At present, there are no local recurrences, and the follow-up observation is continued.

There are still several shortcomings in this clinical study. One, the number of cases, is insufficient, in the follow-up treatment, we can extend the time span of collecting cases and increase the detection rate of early colorectal cancer by our endoscopists. For patients with indications for endoscopic ESD treatment, endoscopic treatment should be performed as much as possible. Two, for epidemics, multi-regional study summarization is more persuasive than traditional methods. Three, setting up or achieving a standardized follow-up process to improve the reliability of follow-up. The results of this clinical retrospective study suggest that strict control of surgical indications, improvement of intraoperative operating skills, attention to postoperative pathological feedback, and close follow-up are the key points to improve the clinical application of ESD.

\section{Acknowledgments}

Funding: None.

\section{Footnote}

Reporting Checklist: The authors have completed the STROBE reporting checklist. Available at http://dx.doi. org/10.21037/jgo-20-393

Data Sharing Statement: Available at http://dx.doi. org/10.21037/jgo-20-393

Conflicts of Interest: All authors have completed the ICMJE uniform disclosure form (available at http://dx.doi. org/10.21037/jgo-20-393). The authors have no conflicts of interest to declare.

Ethical Statement: The authors are accountable for all aspects of the work in ensuring that questions related to the accuracy or integrity of any part of the work are appropriately investigated and resolved. This study was approved by the Ethics Committee of the Second Affiliated Hospital of Nantong University (No. 2019KS095). All procedures performed in this study involving human participants were in accordance with the Declaration of Helsinki (as revised in 2013). Individual consent for this retrospective analysis was waived.

Open Access Statement: This is an Open Access article distributed in accordance with the Creative Commons Attribution-NonCommercial-NoDerivs 4.0 International License (CC BY-NC-ND 4.0), which permits the noncommercial replication and distribution of the article with the strict proviso that no changes or edits are made and the original work is properly cited (including links to both the formal publication through the relevant DOI and the license). See: https://creativecommons.org/licenses/by-nc-nd/4.0/.

\section{References}

1. Dekker E, Tanis PJ, Vleugels JLA, et al. Colorectal cancer. Lancet. 2019;394:1467-80.

2. He L, Wang F, Tian H, et al. The expression profile of RNA sensors in colorectal cancer and its correlation with cancer stages. Transl Cancer Res 2019;8:1351-63. 
3. Ferlitsch M, Moss A, Hassan C, et al. Colorectal polypectomy and endoscopic mucosal resection (EMR): European Society of Gastrointestinal Endoscopy (ESGE) Clinical Guideline. Endoscopy 2017;49:270-97.

4. Raju GS, Lum PJ, Ross WA, et al. Outcome of EMR as an alternative to surgery in patients with complex colon polyps. Gastrointest Endosc 2016;84:315-25.

5. Law R, Das A, Gregory D, et al. Endoscopic resection is cost-effective compared with laparoscopic resection in the management of complex colon polyps: an economic analysis. Gastrointest Endosc 2016;83:1248-57.

6. Fuccio L, Hassan C, Ponchon T, et al. Clinical outcomes after endoscopic submucosal dissection for colorectal neoplasia: a systematic review and meta-analysis. Gastrointest Endosc 2017;86:74-86.e17.

7. Alvarez Herrero L, Pouw RE, van Vilsteren FG, et al. Safety and efficacy of multiband mucosectomy in 1060 resections in Barrett's esophagus. Endoscopy 2011;43:177-83.

8. Purchiaroni F, Costamagna G, Hassan C. Quality in upper gastrointestinal endoscopic submucosal dissection. Ann Transl Med 2018;6:261.

9. Chen T, Zhang YQ, Chen WF, et al. Efficacy and safety of additional surgery after non-curative endoscopic submucosal dissection for early colorectal cancer. BMC Gastroenterol 2017;17:134.

10. Oka S, Tanaka S, Saito Y, et al. Local recurrence after endoscopic resection for large colorectal neoplasia: a multicenter prospective study in Japan. Am J Gastroenterol 2015;110:697-707.

11. Saito Y, Fukuzawa M, Matsuda T, et al. Clinical outcome of endoscopic submucosal dissection versus endoscopic mucosal resection of large colorectal tumors as determined by curative resection. Surg Endosc 2010;24:343-52.

12. Saito Y, Uraoka T, Yamaguchi Y, et al. A prospective, multicenter study of 1111 colorectal endoscopic submucosal dissections (with video). Gastrointest Endosc 2010;72:1217-25.

13. Chen W, Zheng R, Baade PD, et al. Cancer statistics in China, 2015. CA Cancer J Clin 2016;66:115-32.

14. Duggan MA, Anderson WF, Altekruse S, et al. The Surveillance, Epidemiology, and End Results (SEER) Program and Pathology: Toward Strengthening the Critical Relationship. Am J Surg Pathol 2016;40:e94-e102.

15. Cui G, Qi H, Gundersen MD, et al. Dynamics of the IL33/ST2 network in the progression of human colorectal adenoma to sporadic colorectal cancer. Cancer Immunol Immunother 2015;64:181-90.

16. Yamada M, Saito Y, Takamaru H, et al. Long-term clinical outcomes of endoscopic submucosal dissection for colorectal neoplasms in 423 cases: a retrospective study. Endoscopy 2017;49:233-42.

17. Tanaka S, Kashida H, Saito Y, et al. JGES guidelines for colorectal endoscopic submucosal dissection/endoscopic mucosal resection. Dig Endosc 2015;27:417-34.

18. Hong MJ, Kim JH, Lee SY, et al. Prevalence and clinical features of coagulation syndrome after endoscopic submucosal dissection for colorectal neoplasms. Dig Dis Sci 2015;60:211-6.

19. Ogasawara N, Yoshimine T, Noda H, et al. Clinical risk factors for delayed bleeding after endoscopic submucosal dissection for colorectal tumors in Japanese patients. Eur J Gastroenterol Hepatol 2016;28:1407-14.

(English Language Editor: J. Chapnick)
Cite this article as: Yu J, Zhang Y, Qian J. Endoscopic submucosal dissection in the treatment of patients with early colorectal carcinoma and precancerous lesions. J Gastrointest Oncol 2020;11(5):911-917. doi: 10.21037/jgo-20-393 